\section{D01.2 AMONG MEN WHO HAVE SEX WITH MEN, PHARYNGEAL INFECTION CONTRIBUTES MORE TO POPULATION LEVELS OF GONORRHEA TRANSMISSION THAN URETHRAL INFECTION, RECTAL INFECTION, OR BOTH (AGAINST THE MOTION)}

H Hunter Handsfield*. University of Washington, Seattle, USA

10.1136/sextrans-2019-sti.3

Under the renowned transmission paradigm $\mathrm{Ro}=\mathrm{CD}_{\mathrm{C}}$, the reproductive rate of an infection $\left(R_{o}\right)$ may be $\geq 1.0$ - sufficient to sustain or raise prevalence in a defined population - despite a short duration of infectivity (D), if transmission efficiency (B) or the rate of transmission events (c) is sufficiently high. $B$ is substantial for urethral to pharyngeal transmission, and probably modest for pharynx to urethra. But is $ß$ for analingus - for pharyngeal to rectal infection or rectal to pharyngeal - sufficient to sustain prevalent infections at either anatomic site? Is $B$ for kissing high enough to contribute substantially to pharyngeal gonorrhea? Overall, is $B$ for pharyngeal gonococcal infection sufficient to account, directly or indirectly, for half or more of all gonorrhea in men who have sex with men (MSM)? This proposition flies in the face of a century of historical opinion and clinical observation, the anatomy of sex, and available data. As observed by Marcello Truzzi and famously popularized by Carl Sagan, extraordinary claims require extraordinary evidence, a standard not met by observations in a single clinic or metropolitan area or by mathematical models that may not account for confounding factors. While bidirectional transmission by fellatio is well documented and contributes to ongoing transmission in MSM, the transmission efficiency of gonorrhea by kissing, or by analingus for either rectal or pharyngeal infection (or by cunnilingus, in either direction) probably is insufficient to sustain prevalence. Indeed, if anal and vaginal sex magically disappeared as sexual practices, gonorrhea might disappear entirely in exclusively heterosexual men and women and would become uncommon in MSM, including those with high rates of partner change. Reject the proposition!

\section{PL01 - THE ROLE OF GENOMICS IN THE DIAGNOSIS AND MANAGEMENT OF STIS}

Monday, July 15, 2019 8:15 AM - 8:50 AM

\section{PL01.1 THE ROLE OF GENOMICS IN THE DIAGNOSIS AND MANAGEMENT OF STIS}

Helena Seth-Smith*. University Hospital Basel, Department of Clinical Bacteriology/ Mycology, Basel, Switzerland

\subsection{6/sextrans-2019-sti.4}

The diagnosis of sexually transmitted infections (STIs) is a prequel to treatment. The more accurate the diagnosis, the better the treatment that can be offered. From syndromic diagnosis, through species diagnosis, to data on antimicrobial resistance (AMR) mechanisms carried by the infectious agent, higher resolution means improved treatment. The speed at which this information can be gathered is also critical to the successful administration of appropriate treatment and patient compliance. With the continuing global rise in STI diagnoses, the observed danger of diagnostic escape, and the imminence of untreatable gonorrhoea, we need to understand the evolution of STIs and the selection pressures that they are under. Whole genome sequencing can contribute a great deal towards this. Genomics has given us many insights into the lifestyles and evolution of bacterial STIs, over long and short term. Genomic surveillance will always be necessary to decipher population dynamics and to characterise novel strains. Work on genital microbiota and metagenomics can provide information on mixed infections. The pressing question is whether genomics can be used to rapidly develop point of care diagnostic tests, enabling practitioners to provide the most appropriate care for their patients.

Disclosure No significant relationships.

\section{PLO2 - IDENTIFICATION AND MANAGEMENT OF COMPLICATED SYPHILIS}

Monday, July 15, 2019 8:50 AM - 9:25 AM

\section{PL02.1 IDENTIFICATION AND MANAGEMENT OF COMPLICATED SYPHILIS}

Christina Marra*. University of Washington, Seattle, USA

\subsection{6/sextrans-2019-sti.5}

While the course of syphilis can be complicated by involvement of virtually all organ systems, arguably the most difficult forms of complicated syphilis to identify and manage are those that involve the nervous system. In the pre-antibiotic era, all patients with syphilis, regardless of symptoms, underwent a lumbar puncture (LP), and those with cerebrospinal fluid abnormalities received augmented therapy in an effort to prevent subsequent symptomatic neurosyphilis. When penicillin became available, this practice seemed unnecessary. Subsequent reports of symptomatic neurosyphilis after appropriate treatment for early syphilis in persons living with HIV revived LP in neurologically asymptomatic individuals. However, its benefit in terms of morbidity averted is unknown, and recommendations have varied despite lack of data. In this presentation, complicated syphilis is defined as neurosyphilis, ocular syphilis, or otosyphilis. Topics to be discussed include epidemiology, host and bacterial risk factors, screening, the role of LP in management, and treatment.

Disclosure No significant relationships.

\section{PLO3 - ENGAGING INDIGENOUS COMMUNITIES IN HEALTH PROGRAMS}

Monday, July 15, 2019 9:25 AM - 10:00
AM

\section{PL03.1 ENGAGING INDIGENOUS COMMUNITIES IN HEALTH PROGRAMS}

Charlotte Loppie*. University of Victoria, Victoria, Canada

\subsection{6/sextrans-2019-sti.6}

Indigenous peoples and communities experience a disproportionate burden of STBBIs within specific historical, socio- 
political and cultural contexts. To better understand these contexts and promote wellness, it is useful to apply a social determinants of health model, which derives its concepts from Indigenous worldviews, philosophies and values. This model utilizes the tree as a natural metaphor of dynamic and interretated structures and systems, to explore Indigenous peoples' experience of STBBIs, which are influenced by stem, core and root determinants. Stem determinants are proximal conditions that directly influence the acquisition and amelioration of STBBIs, while core determinants (e.g. systems, infrastructure, cultural continuity) influence the development of stem determinants. Deeply entrenched root determinants represent sociopolitical, economic and cultural contexts, including racist ideologies, attempted cultural genocide, and political domination, which have the most profound influence on health because they shape core and stem determinants. In order to address the current challenge of STBBIs among Indigneous peoples, we must explore the relationship between the stem, core and root determinants of Indigenous wellness.

Disclosure No significant relationships.

\section{PL04 - 2019 IUSTI PRESTIGIOUS LECTURE - REVISING STI TREATMENT GUIDELINES IN TIMES OF RAPIDLY EVOLVING ANTIMICROBIAL RESISTANT STIS}

\section{Monday, July 15, 2019 1:45 PM - 2:20 PM}

\section{PL04.1 REVISING STI TREATMENT GUIDELINES IN TIMES OF RAPIDLY EVOLVING ANTIMICROBIAL RESISTANT STIS}

Jørgen Jensen*. Statens Serum Institut, Research Unit For Reproductive Microbiology, Copenhagen, Denmark

\subsection{6/sextrans-2019-sti.7}

Clinical guidelines should be evidence based and prepared after a systematic review of preferably randomised controlled clinical trials. They should include clear grading of the recommendations according to the level of evidence and provide an assessment of the benefits and harms of alternative care options. However, many guideline recommendations are not supported by clinical trials, but rely on expert opinion simply because trials are not available. Even when clinical trials are available, they do not always provide relevant answers. This is particularly the situation when rapidly changing antimicrobial susceptibility make even recent clinical trials outdated and invalid.

The emergence of cephalosporin resistant Neisseria gonorrhoeae resulted in recommendations for dual therapy without clinical trials to document superior cure rates. Such trials would be nearly impossible to conduct, as ceftriaxone alone is still highly efficacious, and thus, treatment failure would be a very rare event. The recommendation for the choice of combination therapy and dosing was based mainly on expert opinion and varied significantly between guidelines. Most recently, the UK BASHH gonorrhoea guideline even moved back to ceftriaxone monotherapy reflecting this uncertainty.

For Mycoplasma genitalium, resistance to first-line azithromycin is well above $50 \%$ in many populations and resistance to second-line moxifloxacin is rapidly increasing, leaving very few available treatment options. This has resulted in expert opinion recommendation that testing for this pathogen should be done only in symptomatic patients, and it has been discussed if the recommendation for test of cure should be limited to patients with persisting symptoms. Such changes in recommendations without real clinical evidence will most likely become more common in the future.

Disclosure JSJ received speaker's fee from Hologic, BD, SpeeDx, and Cepheid and serves scientific advisory board of Roche Molecular Systems, Abbott Molecular, and Cepheid

\section{PL05 - PRIORITIES FOR RESEARCH AND PROGRAMMES FOR TRANS COMMUNITIES}

\section{Monday, July 15, 2019 2:20 PM - 2:55 PM}

\section{PL05.1 PRIORITIES FOR RESEARCH AND PROGRAMMES FOR TRANS COMMUNITIES}

Tonia Poteat* ${ }^{*}$ University of North Carolina Chapel Hill, Department of Social Medicine, Chapel Hill, USA

\subsection{6/sextrans-2019-sti.8}

Trans communities across the world are disproportionately burdened by HIV and STIs. Structural, institutional, social, and individual factors have been implicated as drivers of HIV/STI vulnerability. This plenary presentation will summarize the global epidemiology of HIV/STIs and their multi-level drivers among trans populations. Relationships between stigma, sex work, and co-occurring syndemics of substance use, violence, HIV/STIs will be described. Data on effective behavioral and biomedical interventions to prevent HIV/STI acquisition among transgender adolescents and adult will be reviewed. The impact of genderaffirming medical interventions on HIV/STI acquisition risk will be discussed as well as the latest data on pre-exposure prophylaxis uptake, adherence, and drug-drug interactions. More research is needed on HIV/STI epidemiology among trans masculine and gender non-binary individuals, and gaps remain in basic data from trans communities in sub-Saharan Africa and Eastern Europe/Central Asia. Where epidemiologic data exist, implementation science studies are needed to inform how best to implement and scale up multi-component, high impact, prevention care and treatment interventions that address multilevel barriers to HIV/STI prevention and treatment.

Disclosure No significant relationships. 